\title{
Peripheral neuropathy in the COVID-19 era
}

\section{Panagiotis Zis ${ }^{1, *}$}

${ }^{1}$ Neurology and Clinical Neurophysiology Medical School, University of Cyprus, Nicosia, Cyprus. * takiszis@gmail.com

The term peripheral neuropathy refers to disorders of the peripheral nervous system (PNS) including single and multiple (asymmetric) mononeuropathies, and symmetrical involvement of many nerves (polyneuropathy). Further classification depends on a mixture of phenomenological, neurophysiological, pathological and aetiological parameters. The temporal evolution of symptoms divides polyneuropathy (PN) into acute or chronic. Acute PN e.g. Guillain-Barré syndrome (GBS) is rare but an important entity to recognise because whilst at times severe, it is treatable. Most PN are chronic and usually develop over several months.

Coronavirus disease 2019 (COVID-19) is a contagious disease caused by SARS-CoV-2. Symptoms of COVID-19 are variable, but often include fever, cough, headache, fatigue, breathing difficulties, and loss of smell or taste. Neurological complications have been reported in the context of COVID-19 infection both in the acute and subacute phase, as part of the post-COVID syndrome or as a result of the vaccination against SARS-CoV-2.

In the acute phase, it has been reported that GBS prevalence is 15 cases per 100,000 SARS-CoV-2 infections. Demyelinating GBSs variant in particular is the most prevalent. Although not necessarily directly caused by SARS-CoV2, prolonged stay in ICUs is associated with increased risk of ICU related neuro-myopathy. In the chronic phase, preliminary results of ongoing prospective observational studies suggest that patients suffered from COVID-19 have increased risk of developing mild axonal peripheral neuropathy that shows evidence of length dependency.

Interestingly, it has been reported that - even in the absence of electrophysiologically confirmed PN - 1 in 5 patients reported deterioration of pre-existing neuropathic symptoms or development of new neuropathic symptoms, particularly pain, after COVID-19. Such symptoms tend to persist for months.

Further studies are needed to describe the natural history of the PNS involvement during and after COVID-19 infection. 